\title{
Preceptoria de Enfermagem em Saúde da Família: definindo sua identidade e relevância para o Sistema Único de Saúde
}

\author{
Nursing Preceptors in Family Health: Defining their identity and relevance for Health Unic System \\ Preceptoría en salud de La Famylia: Definiendo su identidad y relevancia para el Sistema Único de \\ Salud
}

\author{
Gabriela Moiçó Azevedo*1, Ândrea Cardoso de Souza², Donizete Vago Daher³, Maristela Magalhães \\ dos Santos Cordeiro ${ }^{4}$
}

Como citar esse artigo. Azevedo, GM; Souza, AC; Daher, DV; Cordeiro, MMS. Preceptoria de Enfermagem em Saúde da Família: definindo sua identidade e relevância para o Sistema Único de Saúde. Revista Pró-UniverSUS. 2019 Jan./Jun.; 10 (1): 166-168.

\section{Resumo}

Objetivos: analisar a importância do preceptor para a formação do residente de enfermagem no Sistema Único de Saúde e conhecer o perfil do profissional preceptor da residência em saúde da família do município do Rio de Janeiro. Método: pesquisa qualitativa do tipo descritiva. Será realizada na rede de atenção básica do município do Rio de Janeiro, onde exista Estratégia Saúde da Família e que seja campo de da residência de enfermagem em saúde da família. Será adotado como critério de inclusão, desenvolver a atividade de preceptor no mesmo cenário por no mínimo o período de um ano e o critério de exclusão será ser preceptor, mas encontrar-se de férias ou licenças durante a pesquisa de campo. Para a coleta de dados, será utilizada a entrevista semiestruturada. Posteriormente, os dados serão tratados por meio da análise temática. A pesquisa foi submetida à apreciação do Comitê de Ética em Pesquisa da Universidade Federal Fluminense e da Secretaria de Saúde do Município do Rio de Janeiro. Resultados esperados: promover uma reflexão do papel do preceptor e de que forma sua prática interfere na formação do residente para o SUS, assim como evidenciar o seu perfil e os métodos pedagógicos que utiliza para auxiliar na formação dos enfermeiros residentes.

Palavras-chave: Estratégia saúde da família, Enfermagem, Preceptoria.

\begin{abstract}
Objectives: To analyze the importance of the preceptor for the formation of the nursing resident in the Unified Health System and to know the profile of the preceptor professional of the family health residence in the city of Rio de Janeiro. Method: qualitative research of the descriptive type. It will be carried out in the basic care network of the municipality of Rio de Janeiro, where there is Family Health Strategy and that is the field of nursing home in family health. It will be adopted as an inclusion criterion, to develop the preceptor activity in the same scenario for at least the one year period and the exclusion criterion will be to be preceptor, but to be on leave or leave during field research. For data collection, the semi-structured interview will be used. Subsequently, the data will be processed through the thematic analysis. The research was submitted to the Research Ethics Committee of the Federal Fluminense University and the Health Department of the Municipality of Rio de Janeiro. Expected results: to promote a reflection on the role of the preceptor and how his practice interferes in the formation of the resident for the SUS, as well as to highlight his profile and the pedagogical methods that he uses to assist in the training of resident nurses. Keywords: Family health strategy, Nursing, Preceptory.
\end{abstract}

\section{Resumen}

Objetivos: analizar la importancia del preceptor para la formación del residente de enfermería en el Sistema Único de Salud y conocer el perfil del profesional preceptor de la residencia en salud de la familia del municipio de Río de Janeiro. Método: investigación cualitativa del tipo descriptivo. Se realizará en la red de atención básica del municipio de Río de Janeiro, donde exista Estrategia Salud de la Familia y que sea campo de la residencia de enfermería en salud de la familia. Se adoptará como criterio de inclusión, desarrollar la actividad de preceptor en el mismo escenario por lo menos el período de un año y el criterio de exclusión será ser preceptor, pero encontrarse de vacaciones o licencias durante la investigación de campo. Para la recolección de datos, se utilizará la entrevista semiestructurada. Posteriormente, los datos serán tratados por medio del análisis temático. La investigación fue sometida a la apreciación del Comité de Ética en Investigación de la Universidad Federal Fluminense y de la Secretaría de Salud del Municipio de Río de Janeiro. Resultados esperados: promover una reflexión del papel del preceptor y de qué forma su práctica interfiere en la formación del residente para el SUS, así como evidenciar su perfil y los métodos pedagógicos que utiliza para auxiliar en la formación de los enfermeros residentes.

Palabras clave: Estrategia salud de la familia, Enfermería, Preceptoria.

Afiliação dos autores:

1Enfermeira. Mestranda do programa de Mestrado Profissional em Ensino na Saúde/MPES, UFF, RJ, Brasil. Email: gabicffc@gamil.com ORCID: https://orcid.org/0000-0002-8941$516 \mathrm{X}$

2Enfermeira. Professora do Programa de Mestrado Profissional em Ensino na Saúde/MPES, UFF, RJ, Brasil. Email: andriacsouza@gmail.com ORCID: https://orcid.org/0000-00026549-8634

3Enfermeira. Professora do Programa de Mestrado Profissional em Ensino na Saúde/MPES, UFF, RJ, Brasil. Email: donizete@predialnet.com.br ORCID: https://orcid.org/00000001-6249-0808

4 Enfermeira. Mestranda do programa de Mestrado Profissional em Ensino na Saúde/MPES, UFF, RJ, Brasil. Email: maristelamagalhaes14@gmail.com ORCID: https://orcid. org/0000-0001-7362-9633 


\section{Introdução}

Com propósito de reordenar o sistema de saúde foi criada a Estratégia de Saúde da Família. Muitos cursos de pós-graduação na modalidade de Residência passaram a ser desenvolvidos neste cenário. Dentre eles está a Residência de Enfermagem em Saúde da Família, que oportuniza ao aluno enfermeiro um aprendizado a partir da experiência no cotidiano dos serviços de saúde. No curso de Residência o aprendizado técnico-científico acontece por meio da orientação de um profissionalpreceptor que atua na Unidade e que está integralmente voltado para o processo ensino-aprendizagem dos alunos residentes, possibilitando maior qualificação da formação em saúde.

A experiência como preceptora e as leituras realizadas nesta área, apontam a ausência de preparação pedagógica dos profissionais de saúde para atuarem como preceptores na Atenção Primária em Saúde APS. Portanto, faz-se necessário pensar estratégias que possam qualificar os profissionais para que atuem como preceptores no Sistema Único de Saúde-SUS.

O despreparo de profissionais na perspectiva do modelo de promoção à saúde despertou a necessidade de transformações na formação em saúde, no momento desarticulada da realidade do serviço, estruturada por uma prática individualista e distante das discussões dos problemas sociais. ${ }^{1}$

É neste sentido que vemos a importância e o papel do Curso de Residência de Enfermagem em Saúde da Família. Por meio de tutores (que são os professores vinculados às Instituições de Ensino- IES) e preceptores (profissionais do serviço) qualificados, a formação em serviço para uma atenção em saúde comprometida com os princípios do SUS, poderá sobremaneira, orientar a formação e ser determinante para a formação de profissionais mais compromissados com um cuidado ético, produtor de vínculo e acolhimento e que tenha centralidade no usuário. Assim, fica evidenciado que a formação do residente deve ser efetivada a partir de uma articulação das IES e os serviços de saúde.

O papel do preceptor na Residência de Enfermagem Saúde da Família vai sendo lapidado e entendido conforme os processos de trabalho vão acontecendo dentro da Unidade e na relação com a equipe. Assim, o preceptor, os profissionais e os residentes vão se qualificando a partir na experiência no cotidiano dos serviços de saúde.

É importante destacar que a preceptoria, enquanto prática educativa é uma atividade que demanda planejamento, competência, criatividade e sensibilidade.

O preceptor é o profissional que participa do processo de formação em saúde ao articular a prática ao conhecimento científico. Ele precisa dominar a prática clínica, bem como os aspectos pedagógicos relacionados a ela, transformando o cenário profissional em ambiente educacional. ${ }^{2}$

No entanto, nem todos os profissionais que atuam como preceptores do SUS possuem formação pedagógica adequada para desempenhar tal função.

Objetivos: analisar a importância do preceptor para a formação do residente de enfermagem no Sistema Único de Saúde; conhecer o perfil do profissional preceptor da residência em saúde da família do município do Rio de Janeiro.

\section{Materiais e Método}

O estudo será realizado por meio de pesquisa de campo, com abordagem qualitativa, tendo como cenário do estudo será composto pelas unidades de saúde do município do Rio de Janeiro que trabalhem com a Estratégia de Saúde da Família, que sejam campo de prática para a Residência de Enfermagem em Saúde da Família.

Os participantes deste estudo serão enfermeiros preceptores de ambos os sexos que atuam em Residências de Enfermagem de Saúde da Família no município do Rio de Janeiro, tendo como critério de inclusão os preceptores que estiverem atuando na função de preceptor no mesmo cenário por pelo menos 1 ano e o critério de exclusão: preceptores que estiverem de férias ou licenças durante a pesquisa de campo.

Para coleta de dados, será utilizada a entrevista semiestruturada que será aplicada de forma presencial aos preceptores da residência de enfermagem em saúde da família, onde responderão a algumas questões norteadoras, abertas, relacionadas com sua prática. O período para a coleta de dados será de três meses, levando em consideração que será preciso visitar várias Unidades de Saúde alocadas em diferentes bairros do município e adequar a data da entrevista ao horário de trabalho e disponibilidade dos profissionais.

Os dados da pesquisa serão tratados por meio da análise de conteúdo temática. Uma análise temática consiste em descobrir os núcleos de sentido que compõe uma comunicação, cuja presença ou frequência significam algo para o objeto analítico escolhido. ${ }^{3}$

Os enfermeiros preceptores a serem entrevistados participarão da pesquisa mediante a assinatura do Termo de Consentimento Livre e Esclarecido, no qual constará o título do projeto, identificação dos responsáveis pelo projeto, o objetivo da pesquisa, os procedimentos necessários à realização e os benefícios que podem ser obtidos, conforme Resolução $n^{\circ} 466 / 12$ do Conselho Nacional da Saúde. Ainda com o objetivo de manter o anonimato dos participantes envolvidos, serão os mesmos, identificados com nomes fictícios.

A pesquisa foi submetida à apreciação do Comitê 
de Ética em Pesquisa com Seres Humanos da UFF e da Secretaria Municipal de Saúde do Rio de Janeiro (SMSRJ).

\section{Resultados esperados}

Espera-se com este estudo promover uma reflexão do papel do preceptor e de que forma sua prática interfere na formação do residente para o SUS, assim como conhecer o seu perfil e os métodos/ estratégias pedagógicas que utiliza.

Planeja-se como produto desta pesquisa a elaboração de uma oficina que seja aplicada a preceptores iniciantes a fim de melhor qualificá-los para o exercício desta importante função, onde será trabalhado métodos pedagógicos para abordagem com o residente. Assim como, a implantação de um espaço na Secretaria Municipal de Saúde- SMSRJ regular de encontro e discussão na modalidade roda de conversa com todos os preceptores do Programa de Residência em Saúde da Família da SMSRJ, com o objetivo de refletirem sobre o seu papel e a forma como se enxergam dentro desta função e quais estratégias e recursos são necessários para o trabalho junto aos residentes. Ao término dos encontros serão enumeradas sugestões do grupo para serem encaminhadas aos tutores, a fim de promoverem maior qualificação das ações e maior articulação entre IES e serviços de saúde.

\section{Referências}

1. Gubert E, Prado ML. Desafios na prática pedagógica na educação profissional em Enfermagem. Rev. Eletr Enf., 2012; 13(2):285-95. Disponível em: <http://www.fen.ufg.br/revista/v13/n2/v13n2a15.htm>. Acesso em: 12 abr. 2017.

2. Botti SHO. Desenvolvendo as competências profissionais dos residentes. Rev Hosp Univ Pedro Ernesto, 2012;11(Supl.1):102-6, 2012. Disponível em: <http://revista. hupe.uerj.br/detalhe_artigo.asp?id=317>. Acesso em: 12 abr. 2017.

3. Bardin L. Análise de conteúdo. 5. ed. Lisboa: Edições 70; Persona, 2010 . 